\title{
How to Differentiate Recurrent Deep Vein Thrombosis from Postthrombotic Syndrome
}

\author{
Gargi Gautam ${ }^{1, *}$ Tim Sebastian²,*(1) Frederikus A. Klok ${ }^{3}$ \\ ${ }^{1}$ Department of Clinical Sciences, Karolinska Institute, Danderyd \\ Hospital, Stockholm, Sweden \\ ${ }^{2}$ Clinic for Angiology, University Hospital Zurich, Zurich, Switzerland \\ ${ }^{3}$ Department of Medicine - Thrombosis and Hemostasis, Leiden \\ University Medical Center, Leiden, The Netherlands \\ Address for correspondence Dr. med. Tim Sebastian, University \\ Hospital Zurich, Rämistrasse 100, 8091 Zürich \\ (e-mail: tim.sebastian@usz.ch). \\ Hämostaseologie 2020;40:280-291.
}

\begin{abstract}
Keywords

- venous thromboembolism

- deep vein thrombosis

- recurrent VTE

- postthrombotic syndrome

Venous thromboembolism (VTE) is associated with significant morbidity and mortality. Accuracy of diagnosis is thus of vital importance. Failure to diagnose VTE increases the risk of progression and complications. Conversely, anticoagulation as a result of an incorrect diagnosis exposes patients to the associated hazards of bleeding. The diagnostic management of recurrent deep vein thrombosis (DVT) and postthrombotic syndrome (PTS) is especially challenging due to the lack of well-established diagnostic standards. Particularly, the differentiation between the two is notoriously difficult as symptoms, clinical signs, and diagnostic test findings largely overlap. This review highlights the current diagnostic and management strategies for recurrent DVT and PTS with a focus on clinical findings and imaging modalities. We also discuss current open questions for clinicians in the field, anticipating future directions and predictions for the year 2050 .
\end{abstract}

\section{Introduction}

Venous thromboembolism (VTE) is a leading cause of cardiovascular morbidity and mortality. It is a composite condition of deep vein thrombosis (DVT) with or without pulmonary embolism. VTE affects 115 to 269 per 100,000 population annually with a pulmonary-embolism-related mortality rate of 6.5 per 100,000 population. ${ }^{1,2}$ The cornerstone in the treatment and prevention of VTE is anticoagulation therapy with its own associated risk of bleeding. . $^{3,4}$

In the absence of provoking factors (e.g., major trauma or surgery), the risk of VTE recurrence after completion of anticoagulation therapy can be as high as $36 \%$ after 10 years with 3 to $4 \%$ of events being fatal. ${ }^{5,6}$ A diagnosis of recurrent VTE after earlier anticoagulant discontinuation usually results in reestablishment of anticoagulation therapy. Since it is often reinstated for an indefinite duration, an incorrect diagnosis

Contributed equally.

received

January 30, 2020

accepted after revision

May 5, 2020

exposes the patient to unnecessary risk of bleeding complications. ${ }^{5-8}$ Management of suspected recurrent VTE is challenging as there is no diagnostic standard. Moreover, pretest probability and imaging techniques used for DVT diagnosis are more unreliable in the setting of suspected recurrence.

In addition, the diagnosis of suspected recurrent DVT is challenging when it involves previously affected vein segments and the posttreatment status (whether initially affected veins recanalized under previous treatment or not) is unknown. The clinical presentation of recurrent DVT can often be more acute with similar signs and symptoms to those of the initial DVT. If symptoms are subacute, it is often difficult to differentiate the clinical presentation of recurrent VTE and postthrombotic syndrome (PTS). 9,10

PTS is a frequent complication after DVT, particularly in the lower extremities: 20 to 50\% of patients will develop PTS within months to few years. ${ }^{10,11}$ PTS represents an important socioeconomic burden and can lead to reduced quality of life and productivity. ${ }^{12}$ The high incidence of PTS complicates the

(c) 2020 Georg Thieme Verlag KG Stuttgart · New York
DOI https://doi.org/

10.1055/a-1171-0486. ISSN 0720-9355. 
assessment of recurrent VTE, since there is a significant overlap in clinical signs and symptoms. Although PTS is regarded as a chronic condition, acute complications such as swelling, inflammation, and venous eczema can suddenly emerge. Less acute symptoms of PTS include pain, chronic edema, heaviness, skin changes, and varicose veins. ${ }^{10,13}$

To date, there is no diagnostic standard for diagnosis of recurrent DVT. Pretest probability and imaging techniques used for DVT diagnosis are less reliable in the setting of suspected recurrence. The resulting uncertainty is critical, since diagnosis of recurrent VTE after anticoagulant discontinuation usually results in its reestablishment. As anticoagulation therapy often is reinstated for an indefinite duration, an incorrect diagnosis exposes the patient to unnecessary risk of bleeding complications, which are both difficult to predict and to prevent. ${ }^{5-8}$

In this review, we will discuss current diagnostic strategies used to diagnose and differentiate recurrent DVT from PTS with the focus on the lower extremities where both conditions are more frequent. ${ }^{14}$ We will discuss novel diagnostic and therapeutic strategies and anticipate future directions and predictions.

\section{Diagnosis of Recurrent Deep Vein Thrombosis}

Patients with suspected recurrence have a higher prevalence of DVT as well as a higher pretest probability than patients with a suspected first DVT episode. ${ }^{9,15-19}$ The latter may be evaluated by clinical decision rules (CDRs) which can be used in combination with a D-dimer test to rule out the diagnosis without evaluation with imaging tests. ${ }^{9}$ In suspected recurrent DVT, the proportion of patients in which this is can be done is lower. Compression ultrasonography (CUS) is the current first-choice imaging tool for DVT. Its interpretation is notably more challenging in patients with suspected ipsilateral recurrence. ${ }^{20-22}$

Studies on recurrent VTE are scarce. In the following paragraphs, we will discuss pretest assessment, laboratory testing, and imaging techniques for suspected recurrent DVT.

\section{Clinical Decision Rules}

CDRs have an important role in the diagnostic management of suspected VTE, particularly in patients classified with low to intermediate risk. They were implemented to address the overuse of diagnostic imaging by using risk stratification to determine which patients to further evaluate. In the Wells score, ${ }^{23}$ the most commonly utilized and studied CDR for DVT, risk factors and clinical examination findings render points between -2 and 9 . The subjective judgment of the clinician is also incorporated where, if an alternative diagnosis is equally or even more likely, two points are reduced. ${ }^{16}$ To improve the diagnostic performance among patients with suspected recurrent DVT, the original Wells score was adjusted by assigning one extra point for the presence of a previous documented DVT. ${ }^{15}$ This automatically moves the risk class from low $(\leq 0$ points) to intermediate (1-2 points) for patients with a history of DVT. A score $\geq 3$ suggests a high risk of DVT. In a meta-analysis by Geersing et al including 10,002 individual patient datasets, the adjusted Wells was sufficiently accurate, whereas the unadjusted score was not. ${ }^{15}$

There have been studies comparing alternative risk scores in various settings, most of which show similar diagnostic performance to the Wells score. ${ }^{24,25}$ Because the Wells score has been validated in large prospective outcome trials, it remains the CDR of choice in the diagnostic management of DVT, although guidelines do not specify a preferred CDR but rather suggest the use of any validated CDR for diagnostic VTE algorithms. ${ }^{22,26}$

To date, no CDR has been exclusively validated in a large cohort of patients with recurrent DVT. Nevertheless, as they were part of the unselected cohorts from which these rules derive, the utilization of $C D R$ is suggested even for suspected recurrent VTE. ${ }^{22,26}$ In a prospective cohort study, including $20 \%$ of patients with a history of previous VTE, a simplified score (I-DVT score) with four parameters was evaluated and was shown to have comparable diagnostic accuracy to the Wells score. ${ }^{27}$ However, this score lacks external validation.

\section{D-Dimer}

Patients with suspected DVT are often subjected to laboratory testing. The results of these tests, in combination with a low probability according to $\mathrm{CDR}$, are used to identify patients where further imaging is required. Most studies on laboratory markers focus on D-dimer, a degradation product of fibrinolysis that is elevated in the blood in the setting of acute thrombosis. Current practice guidelines advise the usage of D-dimer in patients with an unlikely pretest probability, notwithstanding poor levels of evidence. In patients with suspected recurrence, low or unlikely pretest probability, and a below the cutoff high sensitivity D-dimer assay, imaging tests are suggested to be withheld. ${ }^{22,26}$

Several prospective studies have investigated the performance of D-dimer tests, in relation to imaging findings and risk stratification, in patients with suspected recurrent DVT. In a prospective management trial including 300 patients with suspected recurrent DVT, D-dimer testing alone was used to determine the need for further evaluation with CUS. A total of 166 patients had elevated D-dimer, of which 54 (33\%) patients had a positive CUS. In patients with below the cut-off D-dimer, the 3-month incidence for recurrent DVT was estimated to be $6.0 \%$ (95\% confidence interval [CI]; $2.6-11.4 \%$ ), which is considerable. ${ }^{28}$ In a study where ultrasound findings instead were evaluated prior to D-dimer testing, $38(26 \%)$ of 146 patients had a CUS indicative of recurrent DVT, and 108 (74\%) patients with negative CUS underwent further D-dimer testing. D-Dimer was elevated in $31(21 \%)$ patients, and recurrent DVT was confirmed by repetitive or alternative imaging in six (4\%). The remaining were left untreated and the 3-month incidence of recurrent VTE at follow-up was $0 \%(95 \% \mathrm{CI} \text { : } 0-4.8 \%)^{29}$

In the aforementioned meta-analysis by Geersing et al, 941 (9.4\%) patients had suspected recurrence. The failure rate in the subset of these patients with a below the cut-off D-dimer in combination with a low-risk probability by the adjusted Wells score was $1.0 \%$ (95\% CI: $0.6-1.6 \%) .{ }^{15}$ The failure rate of the 
unadjusted Wells score was $2.5 \%$ (95\%CI: $1.2-5.4 \%$ ). Notably, these failure rates need to be assessed in the perspective of the very low DVT prevalence. ${ }^{30}$ Even so, there are several other studies supporting the usage of a combination of a low probability with a below the cut-off D-dimer result to rule out recurrence in patients with suspected recurrent DVT. ${ }^{17,31}$

There have been several suggestions for cut-offs, and more recently, age-adjusted D-dimer levels have been suggested as an improvement to reduce false-positive rates, particularly in the elderly. ${ }^{32}$ In a recent retrospective cohort study, 972 patients were evaluated for DVT. Using age-adjusted cut-offs, specificity was improved while sensitivity levels maintained, and the costs of evaluation were reduced. ${ }^{33}$ Other studies have confirmed these findings. ${ }^{34}$ Nonetheless, prospective studies validating the performance of age-adjusted D-dimer in patients with suspected recurrence and low clinical probability are still required.

\section{Imaging Techniques}

CUS is currently the imaging method of choice in examining suspected DVT in extremities. Limitations of CUS include difficulties in differentiating new thrombi from chronic remains and obtaining reliable reference imaging. . $^{2,35-37}$ There are disparities in the types of examinations used which may complicate the interpretation of guidelines and available studies into clinical practice. Certain centers use a combination of Doppler signal analysis and conventional gray-scale ultrasound (duplex), while others employ sole gray-scale ultrasound strategies. Importantly, certain protocols mandate screening of the entire leg while others recommend scanning only the proximal leg. This is particularly relevant as the treatment spectrum ${ }^{38}$ as well as recurrence risk for isolated calf DVT is different from that for proximal DVT. ${ }^{39,40}$ Additionally, the risk of developing moderate or severe PTS is lower in calf DVT than in proximal DVT, ${ }^{41}$ although still clinically relevant.

Generally, for a first episode of DVT, partial or total incompressibility of a venous segment under gentle pressure from the ultrasound probe is considered diagnostic for thrombosis. ${ }^{42,43}$ When evaluating recurrence it is imperative to consider that postthrombotic changes are present in 50\% of patients 1 year after proximal DVT despite anticoagulant treatment. ${ }^{9,21,40}$ This naturally increases the importance of reference ultrasound examinations to enable accurate diagnosis of recurrence in a previously affected segment.

In the guidance statement document from the International Society on Thrombosis and Haemostasis (ISTH), the main diagnostic criterion for recurrent DVT is the presence of a new, noncompressible venous segment upon CUS. Proximal CUS is recommended as the imaging test of choice. In addition, a follow-up examination at the time of treatment cessation is suggested to obtain baseline reference. Ipsilateral recurrence in a previously abnormal segment is considered diagnosed if the residual vein diameter is $>4 \mathrm{~mm}$. If it is $<2 \mathrm{~mm}$, further imaging tests are suggested only for patients with a high clinical probability. If the diameter is 2 to $4 \mathrm{~mm}$, repeat CUS after 1 week is advised. If the diameter is $>4 \mathrm{~mm}$, treatment initiation is suggested. ${ }^{22}$ Similarly, the more recent American Society of Hematology recommendations also suggest the usage of serial CUS measurements if clinical probability remains high. ${ }^{26}$

Other ultrasound criteria such as echogenicity and thrombus Doppler flow in determining thrombus age and thrombus length measurement for diagnosing recurrence have been evaluated. However, none of them have been validated or used in prospective outcome trials and are thus not included in guideline recommendations. ${ }^{44}$

While CUS-based criteria for ipsilateral recurrence largely rely on reference imaging, there are several obstacles toward a definite diagnosis. First, not all centers perform these examinations routinely. Second, reference reports can be difficult to attain and often contain insufficient information. Last, interpreting the comparison of current with reference images is challenging and interobserver variability is suboptimal.

In a retrospective study by Velthuis and coworkers, comparison with reference examination failed to rule out recurrent DVT in $32 \%$ of cases. ${ }^{21}$ In another study by Linkins et al, 60 patients with previous proximal DVT and high likelihood of residual thrombosis underwent two immediate CUS exams including measurement of residual thrombus diameters. Reproducibility of diameter measurements, as expressed by variance percentage, was $54 \%$ and the average diameter difference between the paired measurements was $2.2 \mathrm{~mm}^{45}$

Previous studies have addressed the clinical implications of residual venous obstruction during follow-up ultrasound to estimate the risk of recurrence and determine whether anticoagulation can be safely stopped. ${ }^{46-49}$ Residual venous obstruction in patients with treated unprovoked DVT was an overall weak predictor for recurrence according to metaanalysis data, ${ }^{47,50}$ while results from studies in specific subsets of patients have shown an increased risk of recurrence..$^{40,51-53}$ In addition to the varying use of reference examinations in clinical practice, there is no consensus for the use of residual vein obstruction measurements in predicting recurrence or a standardized methodology for the measurements. ${ }^{19,22,54}$

In current guidelines, venography and computed tomography (CT) venography are only suggested if CUS is unavailable or results are inadequate. ${ }^{26}$ There are few studies assessing the role of venography in the diagnostic management of recurrent DVT. Thus, there is a clear need for improved imaging methods to incorporate in the diagnostic algorithm for recurrent DVT, in particular when CUS is inconclusive. $^{35-37}$

Magnetic resonance direct thrombus imaging (MRDTI) is currently emerging as a new imaging method in the evaluation of VTE. ${ }^{55-57}$ This method utilizes the presence of methemoglobin, which is formed when hemoglobin in blood clots is oxidized and appears as a high signal upon a T1-weighted magnetic resonance imaging (MRI) sequence (-Fig. 1). ${ }^{58}$ This signal has been shown to disappear completely after 6 months. ${ }^{59}$ In a prospective study, MRDTI scans were performed in 101 patients with confirmed DVT. 

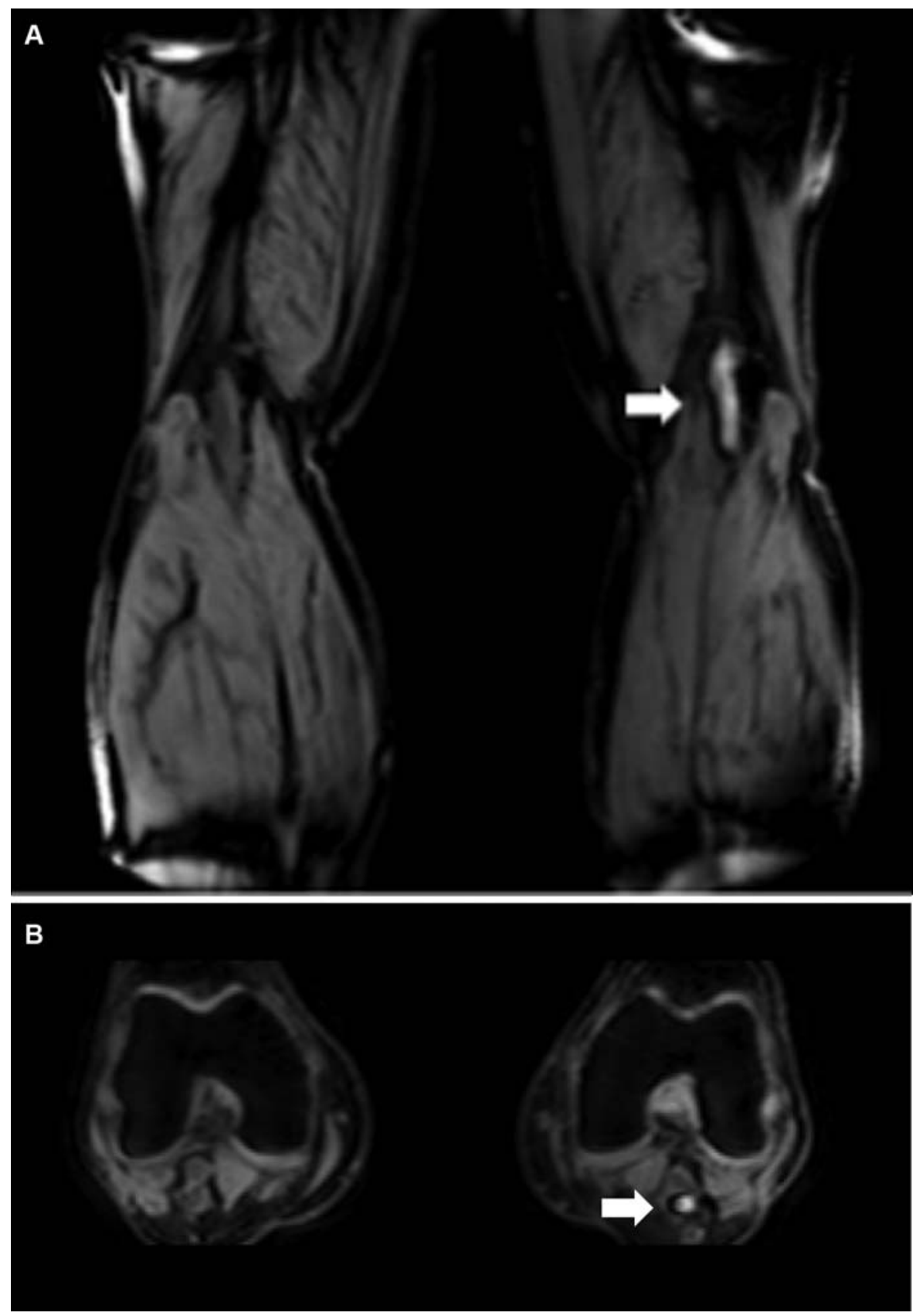

Fig. 1 MRDTI images of a patient presenting with suspected acute symptomatic ipsilateral recurrent DVT. A high signal (indicated by arrows) is present in the left femoral, popliteal, and calf veins. DVT, deep vein thrombosis; MRDTI, magnetic resonance direct thrombus imaging.

MRDTI provided both high sensitivity (97-100\%) and specificity $(100 \%)$ and good interobserver variability (K statistic: $0.89-0.98) .{ }^{58}$ In a subsequent study, 39 patients with confirmed symptomatic recurrent ipsilateral DVT and 42 asymptomatic patients with $\geq 6$ month old chronic residual thrombus remains were examined using MRDTI. Again, the sensitivity (95\% [95\%CI: 83-99\%]), specificity (100\% [95\% CI: 92-100\%]), and interobserver agreement ( $\mathrm{K}$ statistic: 0.98 ) were excellent indicating that MRDTI could be clinically useful to differentiate acute recurrence from residual thrombosis after a previous DVT. ${ }^{60,61}$ As MRDTI has a short acquisition time and is performed without intravenous contrast, ${ }^{58,60}$ it may be a valuable tool in clinical practice, either as a single test or if CUS is unavailable or inconclu- sive. The results of a recent prospective management study are discussed further along in the publication. ${ }^{62}$

\section{Diagnosis of Postthrombotic Syndrome}

Persistent deep venous obstruction causing structural and inflammatory changes of the vein wall and valvular dysfunction is considered to be the main cause of venous congestion and the development of PTS. ${ }^{63}$ The pooling of blood in the leg due to impaired venous return promotes secondary venous insufficiency with reflux distally to the obstruction. The increased venous pressure extents to the level of capillaries where it induces transudation of fluids and proteins causing edema and tissue damage. This is why patients with 
incomplete recanalization after iliofemoral DVT are most likely at risk for PTS whereas infrapopliteal DVT has a lower risk for PTS (hazard ratio [HR]: 1.3; 95\%CI: 1.1-1.6). ${ }^{64,65}$ To date, reliable prediction models have not been established, and there is considerable uncertainty about factors associated with the development of PTS. ${ }^{66,67}$ Suggested risk factors associated with the development of PTS include age, bodymass index (BMI), varicose veins, smoking, residual vein obstruction, sex, provoked DVT, iliofemoral DVT, and recurrent DVT. ${ }^{11,68}$

PTS, recurrent (acute-on-chronic) DVT, and idiopathic chronic venous insufficiency are three common venous disorders that are associated with substantial symptomatic overlap. The latter is a condition not associated with prior DVT. Both PTS and idiopathic chronic venous insufficiency can involve superficial or deep vein reflux, central obstruction, or a combination of both. ${ }^{69}$ Whereas patients with PTS are usually treated with lifestyle modifications or compression stockings, patients with recurrent DVT require anticoagulation therapy, making the distinction between fresh and chronic clots particularly important.

The diagnosis of PTS cannot be made earlier than 3 to 6 months after a DVT as the initial symptoms first must be resolved. ${ }^{10}$ It is primarily made clinically, and several clinical scores are available to assist and standardize the diagnosis. The most commonly used Villalta score consists of five symptoms and six clinical signs which are rated with 0 to 3 points. In the presence of previous DVT, a score of $>4$ points is considered diagnostic for PTS. PTS can be ranked into mild (5-9 points), moderate (10-14 points), or severe (>14 points), which may be helpful to tailor therapy ${ }^{70} \mathrm{~A}$ clinical prediction model, the SOX-PTS score, was recently validated in a cohort of 691 patients with symptomatic proximal DVT, of which $47 \%$ developed PTS. This model includes assessment of the Villalta score, BMI, and anatomical extent of DVT at the time of DVT diagnosis. The authors conclude that the score could be useful in estimating the risk of moderate to severe PTS in particular but further validation is required. ${ }^{68}$

In summary, there is currently no gold standard in the diagnosis and management of PTS. - Fig. 2 shows a proposed clinical flow chart for the management of patients with PTS. For patients with severe PTS and proximal chronic obstruction, angioplasty with stent placement may represent an effective therapeutic option. ${ }^{71}$

\section{Duplex Ultrasound}

Duplex ultrasound is the first-line examination tool to evaluate patency and functionality of the deep venous system. ${ }^{72,73}$ Follow-up duplex ultrasound after DVT may be clinically useful to identify patients at high risk for developing PTS. These patients may be characterized by proximal thrombus residuals and superficial valvular reflux. ${ }^{67} \mathrm{~A}$ lack of recanalization of the common femoral and popliteal vein after 6 months may predict PTS. ${ }^{74}$ In contrast, popliteal reflux after DVT does not appear to be a strong predictor by itself. $^{74,75}$
Parameters used to evaluate the presence of postthrombotic changes by duplex ultrasound encompass morphological and hemodynamic features.

- Gray scale ultrasound (B mode) can be used to visualize degenerative luminal changes of veins, whereas echogenic webs or spurs are distinctive for PTS. In contrast to acute thrombosis, in which the venous lumen expands and is noncompressible, the vein diameter usually decreases in the postthrombotic setting with the lumen being partially compressible, or even disappearing completely. ${ }^{76}$ Presence of large collateral veins, in particular arising from the great saphenous junction which often cross to the contralateral side, may indicate pelvic obstruction.

- Color Doppler mode (C mode) can be used to assess the presence of blood flow in each venous segment and to screen for reflux. Filling defects or complete absence of color signal may indicate venous obstruction.

- Pulsed-wave Doppler (PW mode) signals can be analyzed on the level of flow patterns and peak flow velocities. The PW mode can quantify the extent of venous reflux; the latter is defined as reversed flow for a duration $>1$ second in deep veins or $>0.5$ second in superficial veins. Examination of flow patterns of the common femoral veins can be used as a screening test for iliocaval obstruction; typically in this context it is a monophasic or asymmetrical flow pattern. ${ }^{77}$ Ratios of peak flow velocities can help identify venous stenosis. A poststenotic to prestenotic vein velocity ratio of $>2.5$ in central veins is considered a surrogate marker of significant $\left(>50 \%\right.$ ) lumen stenosis. ${ }^{78,79}$

\section{Cross-Sectional Imaging}

Cross-sectional imaging for PTS includes CT and magnetic resonance venography. Both are helpful to confirm or rule out central venous obstruction at the level of the inferior vena cava or pelvic veins. Whether to perform CT or magnetic resonance venography depends on the local expertise and is not based on clinical practice guidelines. ${ }^{80}$ Both methods require intravenous contrast and are thus associated with risks associated with the use of contrast material and ionizing radiation. Cross-sectional imaging is useful when planning revascularization procedures. If used, duplex ultrasound should always complement the assessment of inguinal veins, because it provides additional hemodynamic parameters to estimate extent of congestion and reflux.

Morphological features of postthrombotic changes observed on CT venography are similar to those on duplex ultrasound ( - Fig. 3). They are characterized by fibrotic bands, decreased venous caliber, luminal obliteration, ipsilateral muscle enlargement, and superficial collateral vein development. ${ }^{81}$ Anatomical abnormalities are frequent findings in patients with previous iliofemoral DVT. Most common is the compression of the left common iliac vein by the right common iliac artery, often along with a bony spur. ${ }^{82}$

Several enhanced or unenhanced protocols exist for magnetic resonance venography. Balanced steady-state free precession pulse sequence is promising for clinical routine because it offers rapid image acquisition without the need 


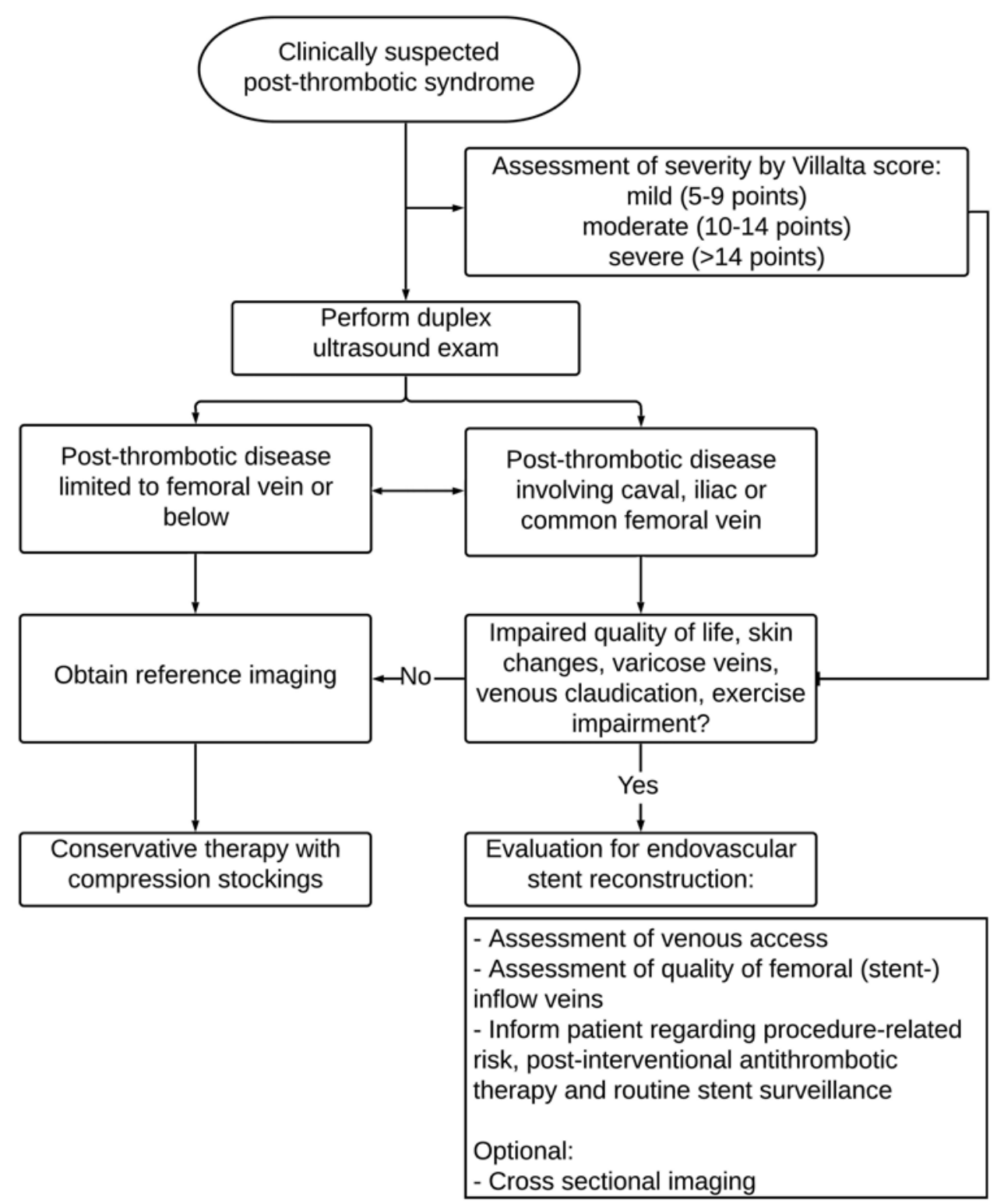

Fig. 2 Flowchart of clinical management suggestions when assessing patients with suspected PTS. PTS, postthrombotic syndrome.

for gadolinium contrast. ${ }^{83}$ As a flow-independent modality, the balanced steady-state free precession pulse sequence is less sensitive to artifact arising from flow variation, in particular respiration. Its performance to determine extent and location of postthrombotic disease was recently validated against contrast venography, and was found to be both sensitive (99\%) and specific (92\%). ${ }^{84}$

\section{Contrast Venography and Intravascular Ultrasound}

Contrast venography is rarely used to diagnose PTS but is applied during endovascular recanalization procedures. Contrast venography is an X-ray examination that uses injected iodinated contrast dye. Multiplanar images are produced to estimate degree of venous stenosis or diagnose occlusion. It has long been the gold standard for diagnosing deep venous obstruction.
Intravascular ultrasound (IVUS) is a catheter-based imaging system composed of a disposable transducer catheter that is attached to a console. Use of IVUS may reduce exposure to ionizing radiation and contrast dye. The catheter is placed into the vein over a wire and produces real-time cross-section images of the vessel. The combination of contrast venography and IVUS has been shown to be more sensitive for identifying and qualifying venous obstruction. ${ }^{85}$

\section{Clinical Management of Recurrent DVT and PTS-Open Questions in 2020}

The Role of MRDTI in the Diagnostic Management of Recurrent Ipsilateral DVT

There is a clear need of improvement of the diagnostic methods available for patients with suspected recurrent DVT, especially in the case of suspected recurrent ipsilateral DVT. This is due to the relatively large proportion of patients 


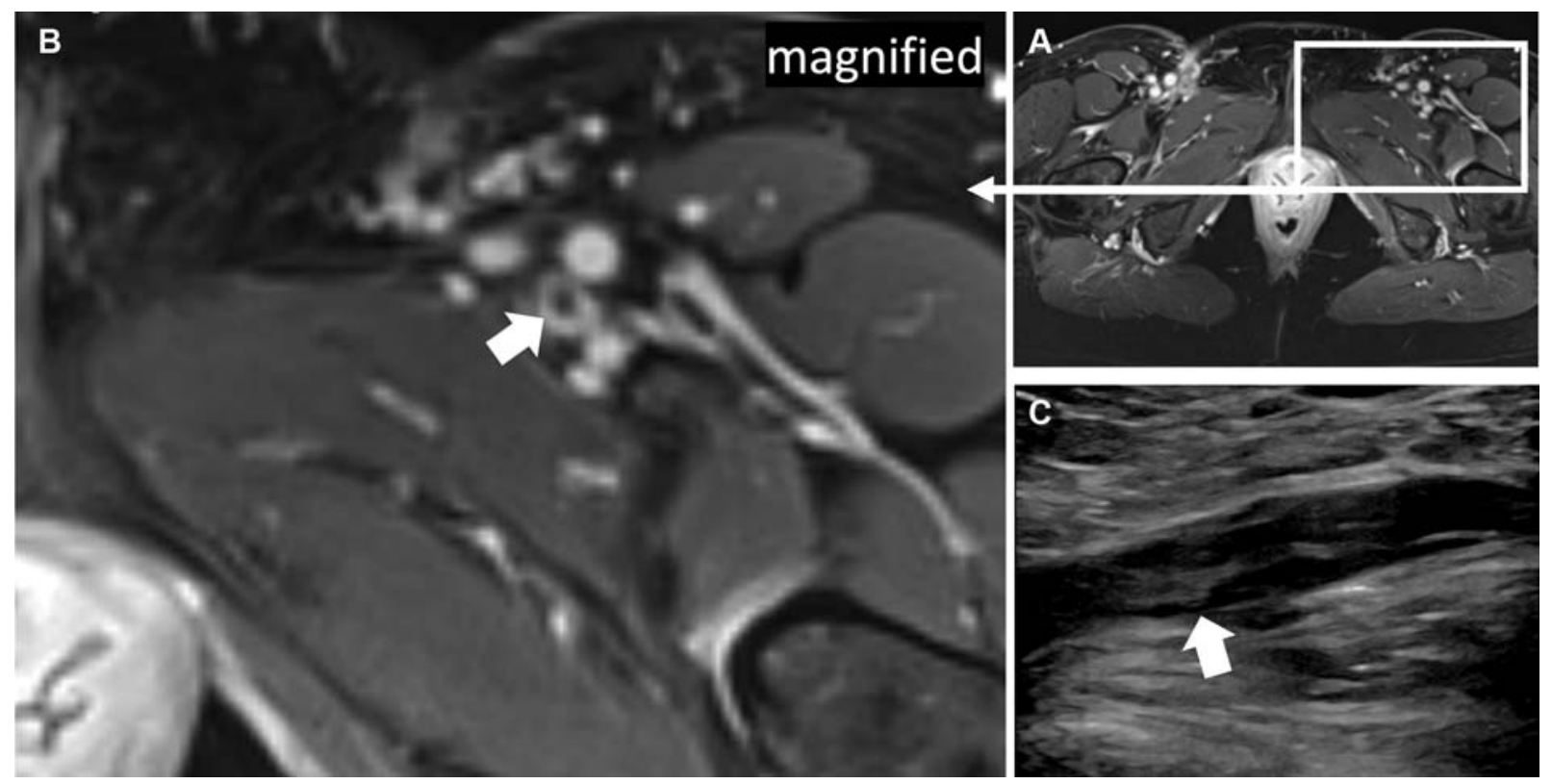

Fig. 3 Postthrombotic changes in the common femoral vein are seen as (A and B) hypodense luminal lesions in MR-venography, and (C) hyperechogenic fibrotic bands in B-mode ultrasound (arrows). MR, magnetic resonance.

with intermediate to high CDR probability in whom D-dimer subsequently cannot be used to rule out recurrence. As previously discussed, there is an unacceptably high proportion of patients where recurrent DVT cannot be ruled out. This often results in prolonged anticoagulant therapy and associated risks. The diagnostic challenges are particularly evident in the management of patients with suspected ipsilateral recurrence of the leg.

The observed potential of MRDTI in differentiating fresh thrombi from chronic thrombotic remains has warranted the need of prospective management studies. ${ }^{26,44}$ In the Theia study, a prospective international multicenter diagnostic management study, 305 consecutive patients were evaluated by MRDTI between 2015 and 2019. Inclusion criteria were (1) suspected acute recurrent ipsilateral DVT where the previous episode was documented, (2) age $\geq 18$ years, (3) ability of the patient to understand the study procedures and their consequences, and (4) signed and dated informed consent prior to the initiation of study procedures. Exclusion criteria were (1) general contraindications for MRI, (2) CUS-proven acute symptomatic DVT within 6 months before current presentation, (3) onset of more than 10 days prior to presentation, (4) suspected acute pulmonary embolism, (5) hemodynamic instability, (6) medical or psychological condition that would not permit completion of the study or signing of informed consent, and (7) noncompliance or inability to adhere to treatment or follow-up visits. All patients were followed up for 3 months. The primary outcome was the 3-month incidence of VTE after an MRDTI examination negative for recurrent DVT. Patients with a negative MRDTI were subjected to a standardized CUS within 48 hours after initial presentation. This examination was not used for the baseline management decision but served as a reference in case the patient returned with symptoms indicative of suspected recurrence during the follow-up period. The incidence of 3-month recurrent VTE among patients with a negative MRDTI was $1.1 \%$ (95\% CI: $0.1-3.8 \%$ ), and the authors suggested that MRDTI can be utilized for therapeutic management decisions in patients with suspected recurrent ipsilateral DVT and inconclusive CUS. ${ }^{62}$

\section{Advanced Treatment Options in PTS}

In addition to improved standardization of the (imaging) diagnosis of PTS, the optimal treatment of PTS is still to be established. When evaluating the therapeutic options for PTS patients, it is important to note the burden of venous claudication, which is most likely underestimated. Venous claudication refers to the development of severe thigh pain and sensation of tightness during exercise affecting up to $40 \%$ of patients after an episode of iliofemoral DVT. ${ }^{86}$ Venous claudication may occur before or even without the manifestation of skin changes, and it is particularly limiting in younger, otherwise healthy, patients. Endovascular therapy has been associated with both ulcer healing and amelioration of venous claudication by restoring venous outflow. ${ }^{87-91}$ For PTS patients with verified chronic venous occlusion, standard compression therapy and angioplasty with provisional stent placement are recommended for those with chronic occlusion of the inferior vena cava or iliac veins who are at risk for venous leg ulcers. ${ }^{92}$

Endovascular therapy has a high technical success and low complication rate. ${ }^{93}$ Although it shows promising clinical outcomes, large outcome studies are unavailable to date and the high incidence of stent thrombosis is an important drawback. In fact, the cumulative incidence of venous stent thrombosis at 6 and 36 months among patients with PTS was $13.7 \%$ (95\%CI: 7.8-19.6\%) and 21.2\% (95\%CI: 13.2-29.2\%), respectively. ${ }^{94}$ Stent thrombosis remains a threat over time and antithrombotic therapy is therefore usually initiated temporarily, or indefinitely after implantation of venous stents. ${ }^{95}$ 


\section{Anticoagulation Therapy after Venous Stent Recanalization}

There is considerable controversy whether all patients with venous stent implants need extended antithrombotic prophylaxis, since PTS patients may not necessarily require such treatment. Since PTS is no life-threatening condition, major bleeding complications are regarded unacceptable in this setting.

To date, there are no randomized-controlled studies available focusing on antithrombotic therapy after the implantation of venous stents. However, there is a general consensus that anticoagulation therapy should be prescribed at least temporarily. ${ }^{69,95}$ However, anticoagulation therapy may not be the key element in preserving patency, and it will not salvage a stent with poor inflow. This is demonstrated by the high incidence of early stent thrombosis which occurs frequently during anticoagulation therapy. ${ }^{94}$ Impaired inflow from the femoral or deep femoral vein has been associated with increased risk for patency loss.

Use of antiplatelet agents in addition to anticoagulation therapy is controversial. ${ }^{69}$ It is under investigation in the international ARIVA (aspirin plus rivaroxaban vs. rivaroxaban alone) study (clinicaltrials.gov; identifier: NCT04128956). Hence, the main indication for long-term anticoagulation in patients with PTS is currently to prevent recurrent DVT.

\section{Stent Surveillance}

Patients with venous stent implants should undergo routine follow-up visits, especially in the early postoperative phase. Stent surveillance by duplex ultrasound can detect abnormalities such as insufficient stent in- or outflow, thrombotic in-stent deposits, or stent fractures jeopardizing long-term success. Early detection of these abnormalities enables the physician to interfere prior to patency loss by reestablishing antithrombotic therapy or performing prophylactic intervention. Stent surveillance is important, since chronic occluded stents are more difficult to treat, and may result in permanent loss of patency with symptom recurrence.

Assessment of patency of venous stents can be challenging, especially in nonfasting or obese patients. To date, no validated criteria exist to diagnose or rule out venous stent obstruction by duplex sonography. In a recent nested case-control study, the performance of various criteria for this prediction was investigated. Flow pattern analysis and peak flow velocities obtained at the stent inlet were both specific and sensitive to diagnose or rule out upstream stent occlusion. However, those criteria appeared to be less sensitive to diagnose stent stenosis, and therefore should always be combined with direct visualization. In the case of poor imaging quality and symptom relapse, only additional imaging (e.g., contrast venography) can reliably exclude venous stent obstruction.

\section{Time Capsule}

- Simplified and modified diagnostic algorithms for patients with suspected recurrent DVT (with consensus for which CDR to use, standardization of the usage of reference imaging, and definition of inconclusive CUS).
- Modified diagnostic algorithm for patients with suspected recurrence on anticoagulant therapy as the indications are increasing, providing additional clinical challenges.

- Improved understanding of the role of extended anticoagulation therapy in the prevention and treatment of PTS.

- Better knowledge of safety and efficacy of endovascular strategies to prevent recurrence and PTS.

\section{Conflicts of Interest}

Dr. Klok reports grants from Bayer, grants from BristolMyers Squibb, grants from Boehringer-Ingelheim, grants from MSD, grants from Daiichi-Sankyo, grants from Actelion, grants from The Dutch Thrombosis Association, grants from The Dutch Heart foundation, outside the submitted work. Dr. Klok reports grants from Bayer, grants from Bristol-Myers Squibb, grants from Boehringer-Ingelheim, grants from MSD, grants from Daiichi-Sankyo, grants from Actelion, grants from The Dutch Thrombosis Association, grants from The Dutch Heart foundation, outside the submitted work. Dr. Sebastian has nothing to disclose. Dr. Gautam has nothing to disclose.

\section{Authors}

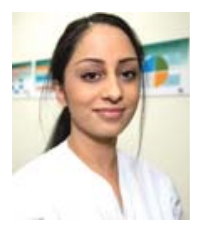

\section{Gargi Gautam}

Gargi Gautam works as a senior resident in Internal Medicine at Danderyd Hospital in Stockholm, Sweden. She combines clinical work in emergency care and coagulation with PhD studies in venous thromboembolism. Her research interests include diagnostic management and recurrence in venous thrombosis.

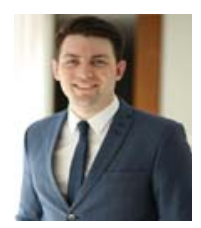

\section{Tim Sebastian}

Tim Sebastian is a resident physician in vascular medicine (angiology) at the University Hospital Zurich in Switzerland. Working under the mentorship of Nils Kucher, he has a special interest in catheter-based therapies including the treatment of deep vein thrombosis and postthrombotic syndrome.

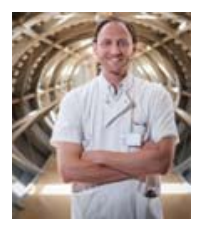

\section{Frederikus A. (Erik) Klok}

Frederikus A. (Erik) Klok is a board-certified specialist in Internal Medicine, with a specialty in vascular medicine in the Leiden University Medical Center (Leiden, the Netherlands) and holds a position as visiting professor at the Center for Thrombosis and Hemostasis in Mainz (Germany). This allows him to combine clinical work with scientific activities. His research interests include the diagnosis, treatment, and long-term complications of venous thromboembolism. 


\section{References}

1 Raskob GE, Angchaisuksiri P, Blanco AN, et al; ISTH Steering Committee for World Thrombosis Day. Thrombosis: a major contributor to global disease burden. Arterioscler Thromb Vasc Biol 2014;34(11):2363-2371

2 Barco S, Mahmoudpour SH, Valerio L, et al. Trends in mortality related to pulmonary embolism in the European Region, 2000-15: analysis of vital registration data from the WHO Mortality Database. Lancet Respir Med 2020;8(03):277-287

3 Klok FA, Kooiman J, Huisman MV, Konstantinides S, Lankeit M. Predicting anticoagulant-related bleeding in patients with venous thromboembolism: a clinically oriented review. Eur Respir J 2015; 45(01):201-210

4 Linkins LA, Choi PT, Douketis JD. Clinical impact of bleeding in patients taking oral anticoagulant therapy for venous thromboembolism: a meta-analysis. Ann Intern Med 2003;139(11):893-900

5 Khan F, Rahman A, Carrier M, et al; MARVELOUS Collaborators. Long term risk of symptomatic recurrent venous thromboembolism after discontinuation of anticoagulant treatment for first unprovoked venous thromboembolism event: systematic review and meta-analysis. BMJ 2019;366:14363

6 van der Wall SJ, van der Pol LM, Ende-Verhaar YM, et al. Fatal recurrent VTE after anticoagulant treatment for unprovoked VTE: a systematic review. Eur Respir Rev 2018;27(150):180094

7 Klok FA, Barco S, Konstantinides SV. External validation of the VTE-BLEED score for predicting major bleeding in stable anticoagulated patients with venous thromboembolism. Thromb Haemost 2017;117(06):1164-1170

8 Klok FA, Hösel V, Clemens A, et al. Prediction of bleeding events in patients with venous thromboembolism on stable anticoagulation treatment. Eur Respir J 2016;48(05):1369-1376

9 Barco S, Konstantinides S, Huisman MV, Klok FA. Diagnosis of recurrent venous thromboembolism. Thromb Res 2018;163:229-235

10 Kahn SR, Comerota AJ, Cushman M, et al; American Heart Association Council on Peripheral Vascular Disease, Council on Clinical Cardiology, and Council on Cardiovascular and Stroke Nursing. The postthrombotic syndrome: evidence-based prevention, diagnosis, and treatment strategies: a scientific statement from the American Heart Association. Circulation 2014;130(18): 1636-1661

11 Ende-Verhaar YM, Tick LW, Klok FA, et al. Post-thrombotic syndrome: short and long-term incidence and risk factors. Thromb Res 2019;177:102-109

12 Caprini JA, Botteman MF, Stephens JM, et al. Economic burden of long-term complications of deep vein thrombosis after total hip replacement surgery in the United States. Value Health 2003;6 (01):59-74

13 Baldwin MJ, Moore HM, Rudarakanchana N, Gohel M, Davies AH. Post-thrombotic syndrome: a clinical review. J Thromb Haemost 2013;11(05):795-805

14 Di Nisio M, van Es N, Büller HR. Deep vein thrombosis and pulmonary embolism. Lancet 2016;388(10063):3060-3073

15 Geersing GJ, Zuithoff NP, Kearon C, et al. Exclusion of deep vein thrombosis using the Wells rule in clinically important subgroups: individual patient data meta-analysis. BMJ 2014;348:g1340

16 Goodacre S, Sutton AJ, Sampson FC. Meta-analysis: the value of clinical assessment in the diagnosis of deep venous thrombosis. Ann Intern Med 2005;143(02):129-139

17 Aguilar C, del Villar V. Combined D-dimer and clinical probability are useful for exclusion of recurrent deep venous thrombosis. Am J Hematol 2007;82(01):41-44

18 Hansson PO, Sörbo J, Eriksson H. Recurrent venous thromboembolism after deep vein thrombosis: incidence and risk factors. Arch Intern Med 2000;160(06):769-774

19 Bates SM, Jaeschke R, Stevens SM, et al. Diagnosis of DVT: Antithrombotic Therapy and Prevention of Thrombosis, 9th ed: American College of Chest Physicians Evidence-Based Clinical Practice Guidelines. Chest 2012;141(2, Suppl):e351S-e418S
20 Tan M, Mos IC, Klok FA, Huisman MV. Residual venous thrombosis as predictive factor for recurrent venous thromboembolism in patients with proximal deep vein thrombosis: a systematic review. Br J Haematol 2011;153(02):168-178

21 Tan M, Velthuis SI, Westerbeek RE, VAN Rooden CJ, VAN DER Meer FJ, Huisman MV. High percentage of non-diagnostic compression ultrasonography results and the diagnosis of ipsilateral recurrent proximal deep vein thrombosis. J Thromb Haemost 2010;8(04):848-850

22 Ageno W, Squizzato A, Wells PS, Büller HR, Johnson G. The diagnosis of symptomatic recurrent pulmonary embolism and deep vein thrombosis: guidance from the SSC of the ISTH. J Thromb Haemost 2013;11(08):1597-1602

23 Wells PS, Hirsh J, Anderson DR, et al. Accuracy of clinical assessment of deep-vein thrombosis. Lancet 1995;345(8961):1326-1330

24 Constans J, Boutinet C, Salmi LR, et al. Comparison of four clinical prediction scores for the diagnosis of lower limb deep venous thrombosis in outpatients. Am J Med 2003;115(06):436-440

25 van der Velde EF, Toll DB, Ten Cate-Hoek AJ, et al. Comparing the diagnostic performance of 2 clinical decision rules to rule out deep vein thrombosis in primary care patients. Ann Fam Med 2011;9(01):31-36

26 Lim W, Le Gal G, Bates SM, et al. American Society of Hematology 2018 guidelines for management of venous thromboembolism: diagnosis of venous thromboembolism. Blood Adv 2018;2(22): 3226-3256

27 Dronkers CE, Tan M, Mol GC, et al. Evaluation of the new simple and objective clinical decision rule "I-DVT" in patients with clinically suspected acute deep vein thrombosis. Thromb Res 2016;141:112-118

28 Rathbun SW, Whitsett TL, Raskob GE. Negative D-dimer result to exclude recurrent deep venous thrombosis: a management trial. Ann Intern Med 2004;141(11):839-845

29 Prandoni P, Tormene D, Dalla Valle F, Concolato A, Pesavento R. Ddimer as an adjunct to compression ultrasonography in patients with suspected recurrent deep vein thrombosis. J ThrombHaemost 2007;5(05):1076-1077

30 Dronkers CEA, Ende-Verhaar YM, Kyrle PA, et al; Subcommittee on Predictive and Diagnostic Variables in Thrombotic Disease. Disease prevalence dependent failure rate in diagnostic management studies on suspected deep vein thrombosis: communication from the SSC of the ISTH. J Thromb Haemost 2017;15 (11):2270-2273

31 Heit JA. Epidemiology of venous thromboembolism. Nat Rev Cardiol 2015;12(08):464-474

32 Nybo M, Hvas AM. Age-adjusted D-dimer cut-off in the diagnostic strategy for deep vein thrombosis: a systematic review. Scand J Clin Lab Invest 2017;77(08):568-573

33 Reardon PM, Patrick S, Taljaard M, et al. Diagnostic accuracy and financial implications of age-adjusted D-dimer strategies for the diagnosis of deep venous thrombosis in the emergency department. J Emerg Med 2019;56(05):469-477

34 Fronas SG, Wik HS, Dahm AEA, et al. Safety of D-dimer testing as a stand-alone test for the exclusion of deep vein thrombosis as compared with other strategies. J Thromb Haemost 2018;16(12): 2471-2481

35 Dronkers CE, Klok FA, Huisman MV. Current and future perspectives in imaging of venous thromboembolism. J Thromb Haemost 2016;14(09):1696-1710

36 Huisman MV, Klok FA. Current challenges in diagnostic imaging of venous thromboembolism. Blood 2015;126(21):2376-2382

37 Huisman MV, Klok FA. Diagnostic management of acute deep vein thrombosis and pulmonary embolism. J Thromb Haemost 2013; 11(03):412-422

38 Robert-Ebadi H, Righini M. Management of distal deep vein thrombosis. Thromb Res 2017;149:48-55

39 Schellong SM, Goldhaber SZ, Weitz JI, et al. Isolated distal deep vein thrombosis: perspectives from the GARFIELD-VTE Registry. Thromb Haemost 2019;19(10):1675-1685 
40 Piovella F, Crippa L, Barone M, et al. Normalization rates of compression ultrasonography in patients with a first episode of deep vein thrombosis of the lower limbs: association with recurrence and new thrombosis. Haematologica 2002;87(05): 515-522

41 Galanaud JP, Righini M, Le Collen L, et al. Long-term risk of postthrombotic syndrome after symptomatic distal deep vein thrombosis: the CACTUS-PTS study.J Thromb Haemost 2020;18(04):857-864

42 Kearon C, Ginsberg JS, Hirsh J. The role of venous ultrasonography in the diagnosis of suspected deep venous thrombosis and pulmonary embolism. Ann Intern Med 1998;129(12):1044-1049

43 Needleman L, Cronan JJ, Lilly MP, et al. Ultrasound for lower extremity deep venous thrombosis: multidisciplinary recommendations from the Society of Radiologists in Ultrasound Consensus Conference. Circulation 2018;137(14):1505-1515

44 Maufus M, Elias A, Barrellier MT, Pernod G; French Society for Vascular Medicine. Diagnosis of deep vein thrombosis recurrence: ultrasound criteria. Thromb Res 2018;161:78-83

45 Linkins LA, Stretton R, Probyn L, Kearon C. Interobserver agreement on ultrasound measurements of residual vein diameter, thrombus echogenicity and Doppler venous flow in patients with previous venous thrombosis. Thromb Res 2006;117(03):241-247

46 Janakiram M, Sullivan M, Shcherba M, Guo S, Billett HH. A systematic review of the utility of residual vein obstruction studies in primary and secondary venous thrombosis. Thrombosis 2013;2013:247913

47 Donadini MP, Ageno W, Antonucci E, et al. Prognostic significance of residual venous obstruction in patients with treated unprovoked deep vein thrombosis: a patient-level meta-analysis. Thromb Haemost 2014;111(01):172-179

48 LE Gal G, Carrier M, Kovacs MJ, et al. Residual vein obstruction as a predictor for recurrent thromboembolic events after a first unprovoked episode: data from the REVERSE cohort study. J Thromb Haemost 2011;9(06):1126-1132

49 Prandoni P, Prins MH, Lensing AW, et al; AESOPUS Investigators. Residual thrombosis on ultrasonography to guide the duration of anticoagulation in patients with deep venous thrombosis: a randomized trial. Ann Intern Med 2009;150(09):577-585

50 Carrier M, Rodger MA, Wells PS, Righini M, LE Gal G. Residual vein obstruction to predict the risk of recurrent venous thromboembolism in patients with deep vein thrombosis: a systematic review and meta-analysis. J Thromb Haemost 2011;9(06):1119-1125

51 Nagler M, Ten Cate H, Prins MH, Ten Cate-Hoek AJ. Risk factors for recurrence in deep vein thrombosis patients following a tailored anticoagulant treatment incorporating residual vein obstruction. Res Pract Thromb Haemost 2018;2(02):299-309

52 Yoo T, Aggarwal R, Wang TF, Satiani B, Haurani MJ. Presence and degree of residual venous obstruction on serial duplex imaging is associated with increased risk of recurrence and progression of infrainguinal lower extremity deep venous thrombosis. J Vasc Surg Venous Lymphat Disord 2018;6(05):575.e1-583.e1

53 Mazetto BM, Orsi FLA, Silveira SAF, et al. Residual vein thrombosis echogenicity is associated to the risk of DVT recurrence: a cohort study. Clin Appl Thromb Hemost 2018;24(03):477-482

54 Watson HG. RVO-real value obscure. J Thromb Haemost 2011;9 (06):1116-1118

55 Dronkers CEA, Klok FA, van Haren GR, et al. Diagnosing upper extremity deep vein thrombosis with non-contrast-enhanced magnetic resonance direct thrombus imaging: a pilot study. Thromb Res 2018;163:47-50

56 Dronkers CEA, Klok FA, van Langevelde K, et al. Diagnosing recurrent DVT of the leg by two different non-contrast-enhanced magnetic resonance direct thrombus imaging techniques: a pilot study. TH Open 2019;3(01):e37-e44

57 Klok FA, Pruefer D, Rolf A, Konstantinides SV. Magnetic resonance direct thrombus imaging for pre-operative assessment of acute thrombosis in chronic thromboembolic pulmonary hypertension. Eur Heart J 2019;40(11):944
58 Fraser DG, Moody AR, Morgan PS, Martel AL, Davidson I. Diagnosis of lower-limb deep venous thrombosis: a prospective blinded study of magnetic resonance direct thrombus imaging. Ann Intern Med 2002;136(02):89-98

59 Westerbeek RE, Van Rooden CJ, Tan M, et al. Magnetic resonance direct thrombus imaging of the evolution of acute deep vein thrombosis of the leg. J Thromb Haemost 2008;6(07):1087-1092

60 Tan M, Mol GC, van Rooden CJ, et al. Magnetic resonance direct thrombus imaging differentiates acute recurrent ipsilateral deep vein thrombosis from residual thrombosis. Blood 2014;124(04): 623-627

61 Klok FA, Tan M, Huisman MV. Letter by Klok et al regarding article, "18F-fluorodeoxyglucose positron emission tomography/computed tomography enables the detection of recurrent same-site deep vein thrombosis by illuminating recently formed, neutrophil-rich thrombus". Circulation 2015;131(24):e530

62 van Dam LF, Dronkers CEA, Gautam G, et al. Diagnosis of suspected recurrent ipsilateral deep vein thrombosis with magnetic resonance direct thrombus imaging. Blood 2020;135(16):1377-1385

63 Bergan JJ, Schmid-Schönbein GW, Smith PD, Nicolaides AN, Boisseau MR, Eklof B. Chronic venous disease. N Engl J Med 2006;355(05):488-498

64 Tick LW, Kramer MH, Rosendaal FR, Faber WR, Doggen CJ. Risk factors for post-thrombotic syndrome in patients with a first deep venous thrombosis. J Thromb Haemost 2008;6(12):2075-2081

65 Labropoulos N, Waggoner T, Sammis W, Samali S, Pappas PJ. The effect of venous thrombus location and extent on the development of post-thrombotic signs and symptoms. J Vasc Surg 2008; 48(02):407-412

66 Amin EE, van Kuijk SMJ, Joore MA, Prandoni P, Ten Cate H, Ten Cate-Hoek AJ. Development and validation of a practical two-step prediction model and clinical risk score for post-thrombotic syndrome. Thromb Haemost 2018;118(07):1242-1249

67 Tick LW, Doggen CJ, Rosendaal FR, et al. Predictors of the postthrombotic syndrome with non-invasive venous examinations in patients 6 weeks after a first episode of deep vein thrombosis. J Thromb Haemost 2010;8(12):2685-2692

68 Rabinovich A, Gu CS, Vedantham S, et al; ATTRACT Trial Investigators. External validation of the SOX-PTS score in a prospective multicenter trial of patients with proximal deep vein thrombosis. J Thromb Haemost 2020;18(06):1381-1389 (e-pub ahead of print). Doi: $10.1111 /$ jth.14791

69 Mahnken AH, Thomson K, de Haan M, O'Sullivan GJ. CIRSE standards of practice guidelines on iliocaval stenting. Cardiovasc Intervent Radiol 2014;37(04):889-897

70 Kahn SR. Measurement properties of the Villalta scale to define and classify the severity of the post-thrombotic syndrome. J Thromb Haemost 2009; 7(05):884-888

71 Ten Cate-Hoek AJ. Prevention and treatment of the post-thrombotic syndrome. Res Pract Thromb Haemost 2018;2(02):209-219

72 Coleridge-Smith P, Labropoulos N, Partsch H, Myers K, Nicolaides A, Cavezzi A. Duplex ultrasound investigation of the veins in chronic venous disease of the lower limbs-UIP consensus document. Part I. Basic principles. Eur J Vasc Endovasc Surg 2006;31(01):83-92

73 Pichot OMC. Role of duplex ultrasound investigation in the management of postthrombotic syndrome. Phlebolymphology 2016;23(02):102-110

74 Prandoni P, Frulla M, Sartor D, Concolato A, Girolami A. Vein abnormalities and the post-thrombotic syndrome. J Thromb Haemost 2005;3(02):401-402

75 Vedovetto V, Dalla Valle F, Milan M, Pesavento R, Prandoni P. Residual vein thrombosis and trans-popliteal reflux in patients with and without the post-thrombotic syndrome. Thromb Haemost 2013;110(04):854-855

76 Hertzberg BS, Kliewer MA, DeLong DM, et al. Sonographic assessment of lower limb vein diameters: implications for the diagnosis and characterization of deep venous thrombosis. AJR Am J Roentgenol 1997;168(05):1253-1257 
77 Kayılıoğlu SI, Köksoy C, Alaçayır İ. Diagnostic value of the femoral vein flow pattern for the detection of an iliocaval venous obstruction. J Vasc Surg Venous Lymphat Disord 2016;4(01):2-8

78 Labropoulos N, Borge M, Pierce K, Pappas PJ. Criteria for defining significant central vein stenosis with duplex ultrasound. J Vasc Surg 2007;46(01):101-107

79 Metzger PB, Rossi FH, Kambara AM, et al. Criteria for detecting significant chronic iliac venous obstructions with duplex ultrasound. J Vasc Surg Venous Lymphat Disord 2016;4(01):18-27

80 Wittens C, Davies AH, Bækgaard N, et al; ESVS Guidelines Committee. Editor's choice - management of chronic venous disease: clinical practice guidelines of the European Society for Vascular Surgery (ESVS). Eur J Vasc Endovasc Surg 2015;49(06):678-737

81 Park EA, Lee W, Lee MW, et al. Chronic-stage deep vein thrombosis of the lower extremities: indirect CT venographic findings. J Comput Assist Tomogr 2007;31(04):649-656

82 Chung JW, Yoon CJ, Jung SI, et al. Acute iliofemoral deep vein thrombosis: evaluation of underlying anatomic abnormalities by spiral CT venography. J Vasc Interv Radiol 2004;15(03):249-256

83 Lindquist CM, Karlicki F, Lawrence P, Strzelczyk J, Pawlyshyn N, Kirkpatrick ID. Utility of balanced steady-state free precession MR venography in the diagnosis of lower extremity deep venous thrombosis. AJR Am J Roentgenol 2010;194(05):1357-1364

84 Helyar VG, Gupta Y, Blakeway L, Charles-Edwards G, Katsanos K, Karunanithy N. Depiction of lower limb venous anatomy in patients undergoing interventional deep venous reconstruction-the role of balanced steady state free precession MRI. Br J Radiol 2018;91(1082):20170005

85 Gagne PJ, Tahara RW, Fastabend CP, et al. Venography versus intravascular ultrasound for diagnosing and treating iliofemoral vein obstruction. J Vasc Surg Venous Lymphat Disord 2017;5(05): 678-687

86 Delis KT, Bountouroglou D, Mansfield AO. Venous claudication in iliofemoral thrombosis: long-term effects on venous hemodynamics, clinical status, and quality of life. Ann Surg 2004;239(01):118-126
87 Delis KT, Bjarnason H, Wennberg PW, Rooke TW, Gloviczki P. Successful iliac vein and inferior vena cava stenting ameliorates venous claudication and improves venous outflow, calf muscle pump function, and clinical status in post-thrombotic syndrome. Ann Surg 2007;245(01):130-139

88 Sebastian T, Dopheide JF, Engelberger RP, Spirk D, Kucher N. Outcomes of endovascular reconstruction of the inferior vena cava with self-expanding nitinol stents. J Vasc Surg Venous Lymphat Disord 2018;6(03):312-320

89 Murphy EH, Johns B, Varney E, Raju S. Endovascular management of chronic total occlusions of the inferior vena cava and iliac veins. J Vasc Surg Venous Lymphat Disord 2017;5(01):47-59

90 Chick JFB, Jo A, Meadows JM, et al. Endovascular iliocaval stent reconstruction for inferior vena cava filter-associated iliocaval thrombosis: approach, technical success, safety, and two-year outcomes in 120 patients. J Vasc Interv Radiol 2017;28(07): 933-939

91 Raju S. Best management options for chronic iliac vein stenosis and occlusion. J Vasc Surg 2013;57(04):1163-1169

92 O'Donnell TF Jr, Passman MA, Marston WA, et al; Society for Vascular Surgery; American Venous Forum. Management of venous leg ulcers: clinical practice guidelines of the Society for Vascular Surgery ${ }^{\circledR}$ and the American Venous Forum. J Vasc Surg 2014;60(2, Suppl):3S-59S

93 Razavi MK, Jaff MR, Miller LE. Safety and effectiveness of stent placement for iliofemoral venous outflow obstruction: systematic review and meta-analysis. Circ Cardiovasc Interv 2015;8(10): e002772

94 Sebastian T, Spirk D, Engelberger RP, et al. Incidence of stent thrombosis after endovascular treatment of iliofemoral or caval veins in patients with the postthrombotic syndrome. Thromb Haemost 2019;119(12):2064-2073

95 Milinis K, Thapar A, Shalhoub J, Davies AH. Antithrombotic therapy following venous stenting: International Delphi Consensus. Eur J Vasc Endovasc Surg 2018;55(04):537-544 